\title{
Narrative Reports to Monitor and Evaluate the Integration of Pharmacists Into Family Practice Settings
}

Kevin Pottie, MD, CCFP, MClSc, FCFP $^{1,2,3}$

Susan Haydt, MA ${ }^{1}$

Barbara Farrell, BScPbm, PharmD $D^{1,3,4}$

Lisa Dolovich, BScPhm, PharmD, $M S_{c}^{5,6,7}$

Connie Sellors, BSc, Pharm ${ }^{6}$

William Hogg, MD, MSc, MClSc $c^{3,8}$

'Élisabeth Bruyère Research Institute,

Ottawa, Ontario, Canada

${ }^{2}$ Institute of Population Health, Ottawa,

Ontario, Canada

${ }^{3}$ Department of Family Medicine at the University of Ottawa, Ontario, Canada

${ }^{4}$ Pharmacy Department, Geriatric Day Hospital at the SCO Health Service,

Ottawa, Ontario, Canada

${ }^{5}$ Centre for Evaluation of Medicines,

McMaster University, Hamilton, Ontario,

Canada

${ }^{6}$ Department of Family Medicine, McMaster University, Hamilton, Ontario, Canada

${ }^{7}$ Leslie Dan Faculty of Pharmacy, University of Toronto, Ontario, Canada

${ }^{8}$ C.T. Lamont Primary Health Care Research Centre, Élisabeth Bruyère Research Institute, Ottawa, Ontario, Canada

Conflicts of interest: none reported.

\section{CORRESPONDING AUTHOR}

Kevin Pottie, MD, CCFP, MClSc, FCFP

1 Stewart St

Ottawa ON K1N 6N5, Canada

kpottie@uottawa.ca

\begin{abstract}
PURPOSE Narratives can capture unfolding events and negotiation of roles and thus can help to evaluate interventions in interdisciplinary health care teams. We describe a practical qualitative method, the narrative report, and its role in evaluating implementation research.
\end{abstract}

METHODS We used narrative reports as a means to evaluate an intervention to integrate pharmacists into group family practices. The pharmacists submitted 63 written narrative reports during a 1-year period. Our interdisciplinary research team analyzed these reports to monitor the progress of the implementation, to identify pharmacists' needs, and to capture elements of the integration process.

RESULTS The monthly narrative reports allowed the research team to document early learning and calibrate the program in terms of clinical support, adapting roles, and realigning expectations. The reports helped the research team stay in tune with practice-related implementation challenges, and the preliminary summary of narrative findings provided a forum for sharing innovations among the integrating pharmacists.

CONCLUSION The narrative report can be a successful qualitative tool to track and evaluate the early stages of an intervention in the context of evolving primary health care teams.

Ann Fam Med 2008;6:161-165. DOI: 10.1370/afm.815.

\section{INTRODUCTION}

$\mathrm{n}$ the IMPACT project (Integrating Family Medicine and Pharmacy to Advance Primary Care Therapeutics), ${ }_{1}^{1}$ we aimed to improve drug ther-

apy using an innovative care model that over a 1-year period integrated nondispensing pharmacists into family practices across Ontario. We implemented this nascent program in 7 group family practices that had 7 to 14 physicians per practice. Although each practice had taken early steps toward working as groups, and 3 had recently introduced a nurse-practitioner, none had developed interdisciplinary teams, nor had they experienced working with a pharmacist as an integrated professional in their practice setting. A key challenge for the IMPACT project was to integrate pharmacists within a family practice setting so they could provide clinical medication-related assessments to physicians, as well as patient education and drug information support.

Primary health care research is often focused on evaluating the initiation, management, and outcomes of change. Writing narratives is a process of detailing and organizing happenings in a cohesive format. The result is a window on the writer's experience, perspective, and negotiation of meaning. Stories serve to make sense of experience, bridge gaps within organizations, and draw attention to the individual. ${ }^{2}$

Diary-style methods have been reported in a range of study fields, 
including care provision studies in nursing, ${ }^{3}$ as well as workplace interaction ${ }^{4}$ and employee innovation processes $^{5}$ in management. Diary-style formats are useful in studies that require information on processes rather than outcomes and provide a means to gather information directly from participants about their experiences on a frequent basis. ${ }^{5}$ These formats are especially useful in capturing cognitive processes related to studies of innovations. ${ }^{5}$ Diaries are also valued for their flexibility; they can range from semistructured formats that record information about a specific event or experience to completely unstructured journal-style formats. ${ }^{3}$

We designed our narrative report to capture stories related to team-based care and the formulation of practice innovations, as both elements were part of the pharmacists' role in the IMPACT study. ${ }^{6}$ The information derived from the narrative reports was part of our program evaluation in that it provided insight into the experiences of the pharmacists who were delivering the program. Such reports allowed us to better understand whether the program implementation was in line with our original assumptions and helped us to interpret the effects of the program on the outcomes under study. ${ }^{7,8}$

In this article we describe a practical method we developed to monitor and evaluate the early stages of implementation as the innovation developed into a stable intervention. Specifically, we describe our design and implementation strategy and the key aspects that encouraged the participants' trust and reflexive responses. Finally, we provide examples to show how our method of using narrative reports helped with program monitoring, support, and calibration during implementation.

\section{METHODS}

The narrative report consisted of a series of questions that aimed to identify pharmacists' observations, struggles, ideas for practice innovation, and successes (a sample report form is shown in the Supplemental Appendix, available online-only at http://www. annfammed.org/cgi/content/full/6/2/161/DC1).

The participants began filing these reports when the intervention was initiated and continued for the first year of implementation. The time log provided subjective information on how the participants spent their time. The report forms were circulated monthly with instructions for completion and return. The Research Ethics Boards of the Élisabeth Bruyère Research Institute and McMaster University approved the study methods.

We provided the participating pharmacists with tips for making practice observations and constructing stories. We encouraged honesty, discouraged businesslike reports, and de-emphasized the need for precise style and grammar. We obtained informed consent from all participants and gave them detailed information outlining how the narrative reports would be used (to improve the intervention, capture emerging innovations, and help develop a road map for future pharmacists integrating into family practice). They also received a description of our de-identification process (all practice sites and names were removed before circulation to the management team) and restricted circulation policy (to management teams and researchers involved with the qualitative analysis). The narrative reports were not circulated among the pharmacists. The management team consisted of 2 project managers and the principal and co-principal investigators. We encouraged participants to use their observation skills, carry a note pad to make notes during the day, and consider using related stories they may be writing to physicians or mentors. As well, we provided sample narrative-type field notes. Further, we provided feedback after each round of reports and a summary of preliminary findings after 4 months, a process outlined by Stone et $\mathrm{al}^{9}$ to maintain retention among participants writing diary-style reports.

The questions remained flexible to allow for creative inquiry and ongoing evaluation of the participants' integration progress. The first 4 months focused on observations, interactions with physicians, and identification of supports and potential innovations. The fifth and sixth months continued in the same manner, but the participants were asked to comment specifically on their developing role and identity as a family practice pharmacist. The seventh month was a member-checking exercise eliciting feedback on a summary of the findings of the first 4 months compiled by the research team. For the eighth narrative participants were asked to comment on their developing relationships with physicians, and for the final narrative they were asked to discuss the tools they believed were necessary to integrate a pharmacist into their particular practice setting based on their experience.

Four members of the research team-1 family physician (K.P.), 2 pharmacists (B.F., N.K.), and 1 sociologist (S.H.) - independently reviewed all monthly narrative reports as they were received. Two additional team members (L.D., C.S.) also read narratives and participated in ongoing discussions. Analysts immersed themselves in the reports, making notes in the margins, writing memos, and assigning codes as themes began to emerge, using immersion/crystallization according to Borkin. ${ }^{10}$ One analyst entered all coding into a qualitative data analysis program (NVIVO) to aid organization. The analysis team (many members also played 
leading roles in program evaluation, project management, and mentor coordination), discussed and debated emerging themes and codes during monthly meetings and suggested supplementary questions for ongoing exploration of areas of interest. The team used an iterative grounded theory ${ }^{11}$ approach to determine common process and content themes related to the pharmacists' integration experience while searching actively for outliers, competing explanations, and meanings. When disagreements persisted, we split coding categories and allowed these to remain distinct from each other. We presented a written report of preliminary findings from the first 4 months to the pharmacists and asked them to comment in a subsequent narrative, and we followed this written member check-up with an in-person focus group of all participating pharmacists.

\section{RESULTS}

The monthly narratives gave the research team a window on the early program implementation process, a process grounded in adaptation and relationship building. Watching the pharmacists adapt and problem solve allowed the research team to document early learning and calibrate the program in terms of clinical support, adapting practice roles, and realigning expectations. This knowledge exchange also helped the management team make decisions about supports, interventions, and individual meetings with pharmacists to assist with the development of the innovation. In the early stages of the project, the pharmacists were concerned about the length of time it took them to prepare the narrative reports. They estimated they spent and average of 1 to 3 hours per month. Although 2 pharmacists continued to find the report burdensome, the remaining 5 adjusted and appreciated the reporting format.

Early in the project the pharmacists' narratives described clinical insecurities, which highlighted the importance of early mentoring, clinical supports, and discussions with practice physicians. As a result, the management team responded with early needs assessments and clinical pharmacy mentoring. Having the pharmacists provide practical clinical recommendations to physicians was a key component of the program. The narratives further allowed the management team to identify issues and emerging innovations from the sites and to focus on and refine solutions that could be shared across the project.

An ongoing issue that I am having problems with is how far to go when doing assessments of patients. It seems that everything that I look at creates more questions. Sometimes the problem is presented as a very simple clinical scenario, but when I look at the patients [sic] charts or talk to the patient, more questions come up. This creates a lot more work and makes me wonder if I should not be just addressing the question at hand (pharmacist 01 ; narrative report 3).

I would like a nice simple assignment from the docs like, "Please check out this patient's compliance for me because her BP is poorly controlled and I have some concern that she might not be taking what I think she is." The pharmacist would be ideally suited for this. I think the docs see the obvious potential but have not realized other potentials (pharmacist 02; narrative report 2).

Early narratives provided a forum for the pharmacists to consider possible system improvements and innovations based on their initial observations of their practice settings. For example, a pharmacist wrote about how to improve the practice's charting system with regard to medication management:

The charts are very unorganized and not up to date at all with respect to what the patient's current medication list is... These unorganized charts can have potential risk for drug therapy monitoring if the physician or nurse cannot find the data. For example, the first 2 patients referred to me were on warfarin and had not had an INR done for over 1 year. As well, 1 of those patients was on digoxin, and her digoxin level was not done for almost 2 years... It would be ideal to have a flow sheet at the front of the chart for warfarin and INR that is always up to date (pharmacist 06; narrative report 1).

The monthly reporting period was also useful for documenting and sharing the progress of implemented office system innovations. Tracking innovations helped the management team identify early successes that could be useful in other settings. The following shows how the narrative tracked the progress of a successful innovation from genesis to completion in a 2 -month period:

[Initial stage] This month ... I've been asked to help out with coming up with some templates for pain assessment/documentation and for diabetes tracking... I'm happy to work on templates for the staff, as I feel that this effort will help their patients and free up some of their time (pharmacist 05, narrative report 4 ).

[Outcome] One thing that has helped enhance practice for the doctors is helping them develop a diabetes tracking flow sheet. This was an initiative that came from them and I've just revised/merged existing flow sheets to suit their needs. I feel that this has gotten most of the physicians talking since it is something that would help their practice (pharmacist 05, narrative report 5).

In addition to documenting these kinds of innovations, pharmacists also used the narratives to record their activities related to patients, including providing education. 
In the last month, I had 3 patients on warfarin, and they did not understand what INR was, what food or drug can interact with warfarin, and why they were going to the lab every few weeks. After I educated them and provided them with the warfarin care booklet and calendar, they had a more clear understanding about the importance of their warfarin (pharmacist 06, narrative report 1).

Writing the narratives helped the pharmacists to clarify their emerging roles to suit their practice setting, to calibrate realistic expectations for the program, and to challenge the implementation process. The pharmacists documented discrepancies between the program's expectations and the physicians' expectations of the pharmacists, as well as what was actually happening in the practices. For example, the IMPACT management team expected computers and other technologies to play a key role, but not all physicians were eager to embrace innovative computer use:

Some of the ideas that have been put forward (to doctors) from IMPACT involve extra efforts on the computer, such as the ideas for sharing more information on prescriptions with the pharmacy. The most computer-savvy doctor in the practice just kind of smiled when I talked to him about this and said that this is very unlikely to go over with the other doctors as many of them are about at the limit of what they are willing to do with the computers (pharmacist 01, narrative report 4).

Conversely, another pharmacist in a different clinic noted a different situation, illustrating the importance of local context when determining the appropriate timing to initiate innovations:

I feel that the physicians have innovations and systems operations as a main priority as opposed to patient assessments. I think the assessments are very much appreciated. But I have the impression that resolving issues on electronic health record may be more useful for them (pharmacist 07, narrative report 4).

The narratives also exposed the pharmacists' growing tension between clinical care and collecting evaluation data for the project. As the pharmacists adapted to the practical work of family practice, their efforts focused on patient-centered activities, while their motivation waned for comprehensive charting and documentation of program process data. Recognizing this stress allowed the research team to try to resolve technical difficulties and streamline data collection as an early priority.

Although I know this is a research project, I find that the documentation and entering data into the database represents a challenge for me. I do what I can since there are still areas that are difficult to use in the database and it is not as streamlined as it could be (pharmacist 05, narrative report 2).
At times, the narratives revealed enthusiasm leading to overextension of the pharmacists. The family practice setting, for example, involved a new set of responsibilities, which presented a challenge for those pharmacists continuing outside jobs already laden with many responsibilities; in such cases, the program team worked to provide personal support and focus tasks.

I feel that I have maxed out my time in all respects (socially and professionally). I do feel stretched between my two positions, and especially the past 2 weeks I have been feeling the "burnout" syndrome settle-in (pharmacist 07, narrative report 4).

\section{DISCUSSION}

The narrative reports provided one means of connecting researchers to the realities of the implementation process and supported early program calibration. The reports helped identify early adaptation challenges, as well as successes, and helped our program management team effectively intervene with clinical mentoring, program management support, and targeted physician engagement. The opportunity for participating pharmacists to review and provide feedback on the preliminary narrative findings report served to build a sense of shared experience among participants and facilitated the sharing of early innovations.

Greenhalgh et $\mathrm{al}^{12}$ conducted a systematic review looking at the adoption of innovation in service delivery. They identified several key elements that facilitate adoption: the relative advantage, complexity, compatibility, trialability, and adaptability of the innovation. Our narrative reports provided a means to enhance innovation implementation by using several of these elements. For example, the variation in physician openness to computer innovation disclosed the importance of appreciating local complexity, the success of the diabetic flow sheet illustrated relative advantage, and the calibration of clinical recommendations and practice innovations characterized the importance of adaptability.

The challenges we encountered by introducing this method were in developing a sense of security for participants to share their stories, training the pharmacist participants to detail their experiences in narrative rather than point-form format, and anticipating the time involved for participants to produce the reports. We recognize that self-censorship may have been occurring, but we hope our attention to negotiating a safe process mitigated this possibility. Developing written narrative skills can be a daunting task. Nevertheless, we found that engaging the participating pharmacists to help improve implementation helped the research team with on-going quality improvement for the program. 
We have several suggestions for others to consider when using narrative reports. To promote creativity, security, and honest writing among participants, provide them with training tools such as sample reports, obtain their informed consent, and explain data security and privacy procedures. For effective data collection and management, determine a reasonable frequency for reporting, keeping in mind the time requirements to write the narratives. As well, develop the most appropriate system to collect data and consider whether electronic tools, such as e-mail or qualitative data analysis programs, will help. Analysts should read reports on an ongoing basis, develop an early strategy for feedback to participants, and plan for supplementary data collection (eg, in-depth interviews) should sensitive but important themes begin to emerge.

In conclusion, primary health care research frequently involves multifaceted interventions taking place in community settings. In such research, process evaluation plays an important role in the interpretation of findings. Practical research methods that meet interrelated monitoring and process evaluation goals, such as narrative summary reports, are worth considering. We found the narrative report an engaging and effective multipurpose research tool when exploring the practitioner role in the development of interdisciplinary primary care teams.

To read or post commentaries in response to this article, see it online at http://www.annfammed.org/cgi/content/full/6/2/161

Submitted June 14, 2007; submitted, revised, October 29, 2007; accepted November 7, 2007.

Key words: Narration; qualitative research; interdisciplinary health team; primary health care; family practice
Funding support: IMPACT was a large-scale demonstration project supported by the Ontario Primary Health Care Transition Fund (20042006). IMPACT Web site: http://www.impactteam.info.

\section{References}

1. Dolovich L, Pottie K, Farrell B, et al. Integrating family medicine and pharmacy to advance primary care therapeutics (IMPACT). Can Pharmacists J. 2004;137(6):22

2. Greenhalgh T, Russell J, Swinglehurst D. Narrative methods in quality improvement research. Qual Saf Health Care. 2005;14(6):443-449

3. Ross MM, Rideout EM, Carson MM. The use of the diary as a data collection technique. West J Nurs Res. 1994;16(4):414-425.

4. Tschan F, Semmer N, Inversin L. Work related and "private" social interactions at work. Soc Indic Res. 2004;67:145-182.

5. Unsworth K, Clegg C. The use of diary methods to study new questions in employee innovation. In: Proceedings, 18th Annual Australian New Zealand Academy of Management Conference. Dunedin, New Zealand: ANZAM; 2004.

6. Integrating Family Medicine and Pharmacy to Advance primary Care Therapeutics (IMPACT) Web site. http://www.impactteam.info/ about.html.

7. Greenhalgh T, Bate P, Macfarlane F, Kyriakidou O. Diffusion of Innovations in Health Service Organisations: A Systematic Literature Review. Malden, MA: Blackwell Publishing; 2005.

8. Weiss C. Evaluation: Methods for Studying Programs and Policies. 2nd ed. Upper Saddle River, NJ: Prentice Hall; 1998.

9. Stone AA, Kessler RC, Haythornwaite JA. Measuring daily events and experiences: decisions for the researcher. J Pers. 1991;59(3):575-607.

10. Borkin J. Immersion/crystallization. In Crabtree BF, Miller WL, eds. Doing Qualitative Research. 2nd ed. Thousand Oaks, CA: Sage Publications; 1999:179-194.

11. Addison RB. (1999). A grounded hermeneutic editing process. In Crabtree BF, Miller WL, eds. Doing Qualitative Research. 2nd ed. Thousand Oaks, CA: Sage Publications; 1999:145-161.

12. Greenhalgh T, Robert G, Macfarlane F, Bate P, Kyriakidou O. Diffusion of innovations in service organisations: Systematic review and recommendations. Milbank Q. 2004;82(4):581-629. 